\title{
Upregulation of SNHG12 accelerates cell proliferation, migration, invasion and restrain cell apoptosis in breast cancer by enhancing regulating SALL4 expression via sponging miR-15a-5p
}

\author{
J. H. YUAN ${ }^{1}$, W. X. $\mathrm{LI}^{1}$, C. HU², B. ZHANG ${ }^{3, *}$ \\ ${ }^{1}$ Department of General Surgery, Hanyang Hospital Affiliated to Wuhan University of Science and Technology, Wuhan, Hubei, China; ${ }^{2}$ Depart- \\ ment of Nursing, Hanyang Hospital Affiliated to Wuhan University of Science and Technology, Wuhan, Hubei, China; ${ }^{3}$ Second Department of \\ Breast Tumor, Pu'ai Campus, Central Hospital of Huangshi, Huangshi, Hubei, China
}

${ }^{*}$ Correspondence: mojbee@163.com

Received August 8, 2019 / Accepted November 13, 2019

\begin{abstract}
Breast cancer $(\mathrm{BC})$ is malignant cancer that threatens the health of millions of females worldwide. Long non-coding RNA (lncRNA) small nucleolar RNA host gene 12 (SNHG12) has been identified as an oncogene in multiple cancers. However, the regulatory role of SNHG12 in BC cell progression is still obscured. The levels of SNHG12, miR-15a-5p, and Sal-like 4 (SALL4) in BC tumor tissues and cells were measured by qRT-PCR. Cell viability, apoptosis, migration, and invasion were examined by CCK8, flow cytometry, and transwell assay, respectively. The interaction between miR-15a-5p and SNHG12 or SALL4 was evaluated by dual-luciferase reporter assay. Protein expression of SALL4 was analyzed by western blot. Xenograft mice were established by subcutaneously injecting BC cells stably transfected with sh-SNHG12 and sh-NC. SNHG12 and SALL4 expressions were upregulated whereas miR-15a-5p was downregulated in BC tumors compared with normal tissues. Besides, miR-15a-5p was correlated with SNHG12 and SALL4 inversely as calculated by Pearson's correlation coefficient. More importantly, SNHG12 knockdown attenuated BC tumor growth in vitro and in vivo. Subsequently, dual-luciferase reporter assay confirmed the interaction between miR-15a-5p and SNHG12 or SALL4. The rescue experiments revealed that miR-15a-5p inhibitor restored SNHG12 silencing induced inhibition on BC cell proliferation, migration, invasion, and promotion of apoptosis. Additionally, SNHG12 was found to accelerate BC cell progression by absorbing miR-15a-5p to enhance SALL4 expression. SNHG12 promotes cell proliferation, migration, and invasion but suppresses apoptosis in BC by upregulating SALL4 expression via sponging miR-15a-5p, representing potential targets for the development of novel diagnosis and treatment methods.
\end{abstract}

Key words: SNHG12, miR-15a-5p, SALL4, progression, breast cancer

Breast cancer (BC), a malignant non-cutaneous tumor with extremely high morbidity and mortality annually, has threatened the health of millions of females worldwide $[1,2]$. Generally, BC is classified by the receptors, including human epidermal growth factor receptor-2 (HER-2), estrogen (ER), and progesterone receptor (PR) $[3,4]$. Despite great efforts have been made, there were still more than 500,000 deaths globally due to metastasis, recurrence, and poor prognosis [5-7]. The investigation of BC genetically could benefit the understanding of the pathogenesis of $\mathrm{BC}$.

Long non-coding RNAs (lncRNAs) are evolutionarily conserved transcripts with a limited protein-encoding capacity [8, 9]. LncRNAs were originally recognized as transcriptional "noises" but numerous studies indicated that they are involved in diverse physiological and pathological processes, including cell cycle, proliferation, inflammation, invasion, metastasis, apoptosis, and autophagy [10-12]. In recent years, SNHG12, which mapped on chromosome 1p35.3, has been widely accepted as a vital biomarker in a variety of diseases [13]. For example, SNHG12 functioned as an autophagy inducer for neuroprotection in vitro and in vivo following cerebral ischemia and reperfusion injury [14]. In ischemic stroke, SNHG12 served as a competing endogenous RNA (ceRNA) to expedite angiogenesis and recovery by sponging stroke to elevating vascular endothelial growth factor (VEGF) expression [15]. Therefore, we assumed that the dysregulation of SNHG12 might be involved in the pathogenesis of $\mathrm{BC}$.

MicroRNAs (miRNAs) refer to single-stranded RNAs that regulate different biological behavior by interacting with the 
specific messenger RNA (mRNA) and altering gene expression at the post-transcriptional level, including transcription, splicing, and translation [16-18]. Previous studies have implicated that post-transcriptional regulator miR-15a-5p is closely correlated with cell proliferation, survival, and apoptosis. Ectopic expression of miR-15a-5p was observed in many diseases, such as diabetes, osteoarthritis, and cancers [19-21]. For example, the upregulation of miR-15a-5p retarded the atherosclerotic inflammatory response as well as improved the arterial injury of diabetic rats via targeting fatty acid synthase (FASN) [22]. Excess expression of miR-15a-5p induced cell growth and attenuated apoptosis of osteoarthritis chondrocytes through suppressing vascular endothelial growth factor A (VEGFA) [23]. In addition, miR-15a-5p was reported to inhibit peritoneal mesothelial cell inflammation and fibrosis induced by peritoneal dialysis by regulation of VEGFA. However, the role of miR-15a-5p in the malignancy of BC is obscure.

In our present study, we attempted to excavate the molecular mechanism of SNHG12 in tumorigenesis and progression of BC. Our results indicated that SNHG12 facilitated proliferation, migration, invasion and inhibited the apoptosis of BC by upregulating of SALL4 through sponging miR-15a-5p, providing novel biomarkers for $B C$ therapy.

\section{Patients and methods}

Patient tissues. Fresh BC tumor tissues and the matched normal tissues were obtained by surgery from BC patients recruited from Hanyang Hospital Affiliated to Wuhan University of Science and Technology. Those patients have not received preoperative treatments, such as chemotherapy and radiotherapy. The patients have signed informed consents. Our experiments were approved by the Ethics Committee of Hanyang Hospital Affiliated to Wuhan University of Science and Technology.

Cell transfection. BC cell lines MDA-MB-231, MCF-7 and human breast epithelial cells MCF-10A were purchased from ATCC (Manassas, VA, USA) and cultured in DMEM medium (Gibco, Carlsbad, CA, USA) containing 10\% FBS and $0.05 \%$ penicillin/streptomycin. Small interfering RNA (siRNA) targeting SNHG12 (si-SNHG12\#1, si-SNHG12\#2, and si-SNHG12\#3), small hairpin RNA (shRNA) targeting SNHG12 (sh-SNHG12), siRNA negative control (si-NC), shRNA negative control (sh-NC) and pcDNA-SNHG12 were synthesized by Genepharma (Shanghai, China). miR-15a-5p mimics, miR-15a-5p inhibitor (anti-miR-15a-5p), miRNA negative control (miR-NC), and miRNA negative control inhibitor (anti-NC) were purchased from RIBOBIO (Guangzhou, China). All the vectors were transfected into MDA-MB231 and MCF-7 cells using Lipofectamine 2000 (Invitrogen, Carlsbad, CA, USA).

Quantitative real-time polymerase chain reaction (qRT-PCR). BC tumor tissues and cells were lysed by
TRIzol reagent (Invitrogen) to obtain total RNA. The complementary DNA (cDNA) for SNHG12, miR-15a-5p, and SALL4 was synthesized by All-in-One ${ }^{\mathrm{mex}}$ First-Strand cDNA Synthesis Kit (FulenGen, Guangzhou, China). Next, qRT-PCR was performed using SYBR green (Applied Biosystems, Foster City, CA, USA). The expression of SNHG12, miR-15a-5p, and SALL4 was normalized by U6 or GAPDH. The primers for SNHG12, miR-15a-5p, SALL4, U6, and GAPDH were listed as follows: SNHG12, (Forward, 5'-TCTGGTGATCGAGGACTTCC-3'; Reverse, 5'-ACCTCCTCAGTATCACACACT-3'); miR-15a-5p, (Forward, 5'-UAGCAGCACAUAAUGGUUUGUG-3'; Reverse, 5'-CAAACCAUUAUGUGCUGCUAUU-3'); SALL4, (Forward, 5'-AACGCCACTGTCTCCAAGAT-3'; Reverse, 5'-AAGCAGCATAGCAACAATCG-3'); U6, (Forward, 5'-ACCCTGAGAAATACCCTCACAT-3'; Reverse, 5'-GACGACTGAGCCCCTGATG-3'); GAPDH, (Forward, 5'-TCTACATGTTCCAGTATGACTC-3'; Reverse, 5'-ACTCCACGACATACTCAGCACC-3').

CCK-8, flow cytometry, and transwell assay. Cell proliferation, migration, invasion, and apoptosis were measured by CCK-8, transwell, and flow cytometry, respectively. For cell viability detection, transfected MDA-MB-231 and MCF-7 cells (1000 cells/well) were seeded in 96-well plates for $12 \mathrm{~h}$, $24 \mathrm{~h}, 48 \mathrm{~h}, 72 \mathrm{~h}$, and $96 \mathrm{~h}$. Then, CCK-8 reagent (10 $\mu \mathrm{l} /$ well, Beyotime, Shanghai, China) was added for $2 \mathrm{~h}$. The OD value at $450 \mathrm{~nm}$ was measured using a spectrophotometer (Thermo Fisher Scientific, Waltham, MA, USA). For flow cytometry, MDA-MB-231 and MCF-7 cells were collected at $48 \mathrm{~h}$ posttransfection, stained with Annexin V-FITC/PI and counted by BD FACS Canto II flow cytometry (BD Biosciences, Franklin Lakes, NJ, USA). For transwell invasion assay, transfected MDA-MB-231 and MCF-7 cells were placed on the upper chamber pre-treated with Matrigel (without Matrigel treatment for migration assay; Becton Dickinson, Franklin Lakes, NJ, USA). Subsequently, the cells at the lower chamber were stained with $0.1 \%$ crystal violet (Sigma, St. Louis, MO, USA) for $10 \mathrm{~min}$. The migration and invasion cell number was counted using a microscope.

Western blot. Western blot assay was performed following the standard protocol. The primary antibody against SALL4 was purchased from Abcam (Cambridge, MA, USA) and HRP-conjugated secondary antibody was obtained from Sangon (Shanghai, China). Protein bands were visualized by enhanced chemiluminescence reagents (Millipore, Temecula, California, USA) and protein quantification was carried out by ImageJ software (National Institutes of Health, Bethesda, MD, USA).

Dual-luciferase reporter assay. Wild type (WT SNHG12, WT SALL4) and mutant type (MUT SNHG7, MUT SALL4) luciferase vectors were constructed. MDA-MB-231 and MCF-7 cells were co-transfected with those vectors and miR-15a-5p mimics or miR-NC for $24 \mathrm{~h}$ using Lipofectamine 2000 transfection reagent. Luciferase activities were evaluated by dual-luciferase reporter assay. 
RNA immunoprecipitation (RIP). MDA-MB-231 and MCF-7 cells were lysed by RIP buffer and the cell lysates were incubated with magnetic beads coated with anti-Ago 2 or IgG antibody (Millipore). The enrichment of SNHG12 and miR-15a-5p was analyzed by qRT-PCR.

Murine xenograft assay. Female nude mice (4-5 weeks old) were purchased from Vital River Laboratory Animal Technology (Beijing, China). The mice were randomly divided into 2 groups: sh-SNHG12 $(n=6)$ and sh-NC $(n=6)$ group. MDA-MB-231 cells, stably transfected with a lentivirus vector containing sh-SNHG12 or sh-NC, were subcutaneously injected in mice to establish mice model. Tumor volume was measured every 5 days. The mice were sacrificed at day 30 and tumors were collected for biological analysis. Tumor weight was measured when the mice were sacrificed. Animal experiments were approved by the National Animal

Table 1. Relationship between expression of SNHG12 and clinicopathological features of breast cancer.

\begin{tabular}{|c|c|c|c|c|}
\hline \multirow{2}{*}{ Characteristics } & \multirow{2}{*}{$\mathbf{n}$} & \multicolumn{2}{|c|}{ SNHG12 } & \multirow{2}{*}{ p-value } \\
\hline & & High & Low & \\
\hline \multicolumn{5}{|l|}{ Age (years) } \\
\hline$\geq 60$ & 68 & 37 & 31 & \multirow{2}{*}{0.271} \\
\hline$<60$ & 22 & 9 & 13 & \\
\hline \multicolumn{5}{|l|}{ Tumor size $(\mathrm{cm})$} \\
\hline$\geq 3$ & 51 & 32 & 19 & \multirow{2}{*}{$0.012^{\star *}$} \\
\hline$<3$ & 39 & 14 & 25 & \\
\hline \multicolumn{5}{|l|}{ TNM stage } \\
\hline I-II & 30 & 11 & 19 & \multirow{2}{*}{0.053} \\
\hline III-IV & 60 & 35 & 25 & \\
\hline \multicolumn{5}{|c|}{ Lymph node metastasis } \\
\hline Yes & 56 & 36 & 20 & \multirow{2}{*}{$0.001^{* *}$} \\
\hline No & 34 & 10 & 24 & \\
\hline \multicolumn{5}{|c|}{ Distant metastasis } \\
\hline Yes & 11 & 8 & 3 & \multirow{2}{*}{0.126} \\
\hline No & 79 & 38 & 41 & \\
\hline \multicolumn{5}{|l|}{ Vascular invasion } \\
\hline Yes & 37 & 25 & 12 & \multirow{2}{*}{$0.009^{* *}$} \\
\hline No & 53 & 21 & 32 & \\
\hline
\end{tabular}

Care and Ethics Institution and Hanyang Hospital Affiliated to Wuhan University of Science and Technology.

Statistical analysis. All the experiments were repeated at least 3 times and data were presented as means \pm standard deviation (SD). Data analysis was carried out using SPSS software (SPSS, Chicago, Illinois, USA) and GraphPad Prism 7 (GraphPad Inc., San Diego, CA, USA). The correlation between miR-15a-5p and SNHG7 or SALL4 was analyzed by Pearson's correlation coefficient. The survival curve of patients was generated by the Kaplan-Meier plot and analyzed by the log-rank test. A p-value less than 0.05 $(p<0.05)$ was considered statistically significant.

\section{Results}

Upregulation of SNHG12 in BC. SNHG12 levels in BC tumor tissues and the corresponding normal tissues were evaluated by qRT-PCR to assess the importance of SNHG12 in BC cell development. As illustrated in Table 1, SNHG12 was highly expressed in patients with the following features: large tumor size, advanced stage, lymph node metastasis, and vascular invasion. However, SNHG12 was lowly expressed in patients with distant metastasis. The expression of SNHG12 was extremely higher in BC tumor tissues than that of the corresponding normal tissues (Figure 1A). Consistently, SNHG12 level was upregulated markedly in BC cell lines (MDA-MB-231, MCF-7) compared with human breast epithelial cells MCF-10A (Figure 1B). As expected, we discovered that a high level of SNHG12 contributed to a low survival rate in $\mathrm{BC}$ patients. By contrast, a low level of SNHG12 resulted in a high survival rate with 60 months (Figure 1C). All the data indicated that SNHG12 might function as an oncogene in BC.

SNHG12 depletion suppressed proliferation, migration, invasion, and promoted apoptosis of BC cells. The influence of SNHG12 on BC cell progression was further detected using CCK-8, flow cytometry, and transwell assay. MDA-MB-231 and MCF-7 cells were transfected with si-NC, si-SNHG12\#1, si-SNHG12\#2 or si-SNHG12\#3, and the transfection efficiency was measured by qRT-PCR. As
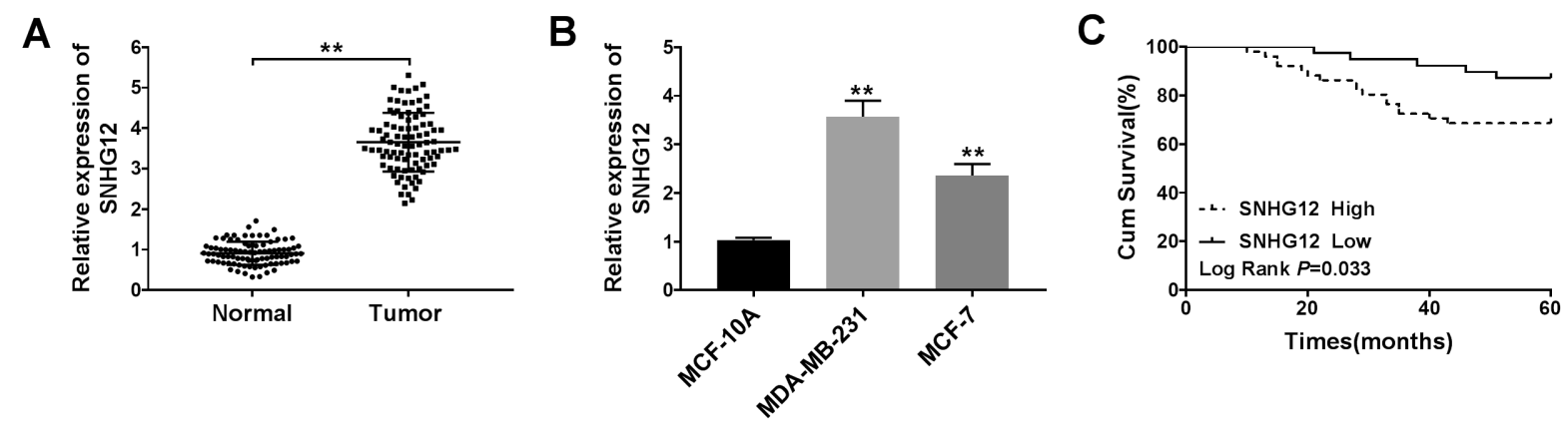

Figure 1. SNHG12 was upregulated in BC tissues and cell lines. A) SNHG12 expression in BC tumor tissues compared with the matched normal tissues. B) SNHG12 expression in BC cell lines (MDA-MB-231, MCF-7) compared with human breast epithelial cells MCF-10A. C) The survival rate of BC patients with high and low levels of SNHG12. ${ }^{* *} \mathrm{p}<0.01$ 
A

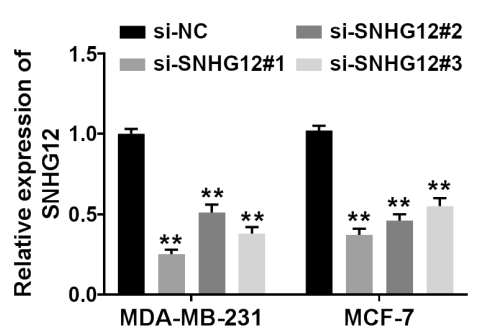

D
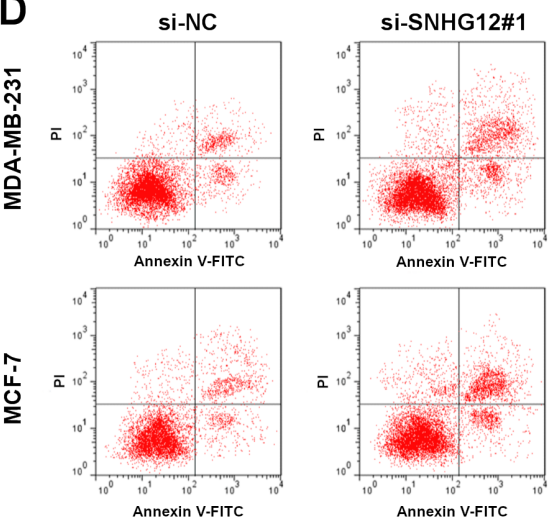

E

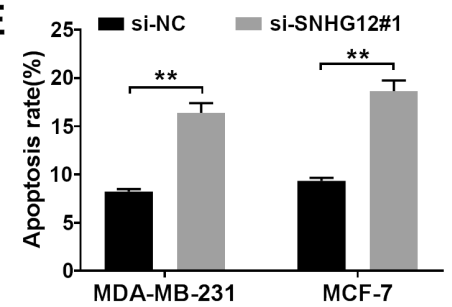

B
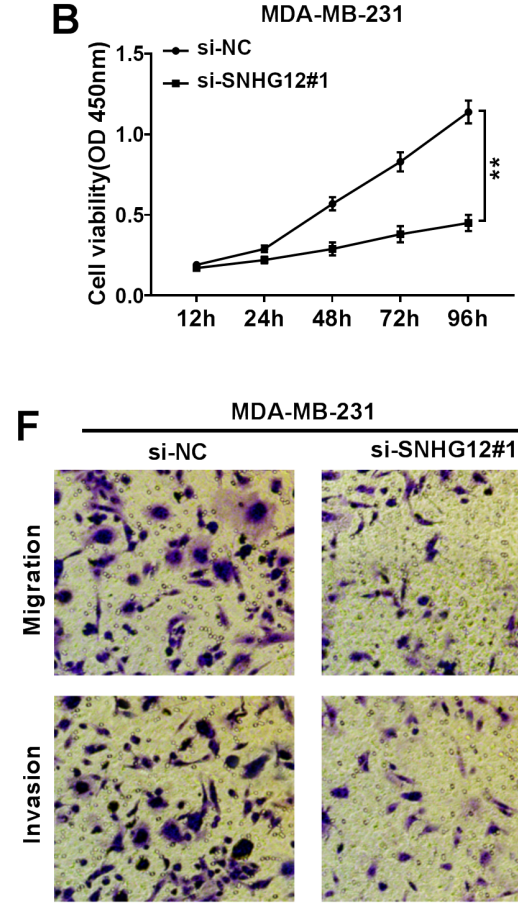

H

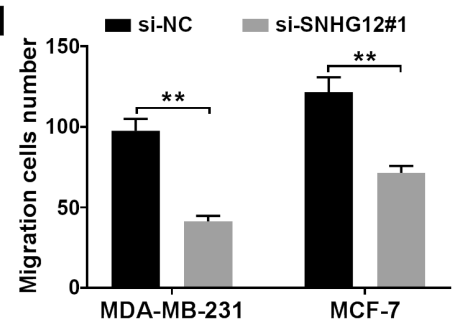

C

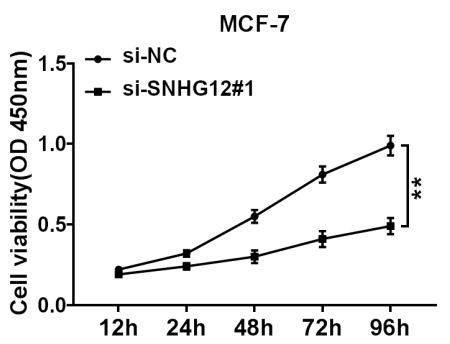

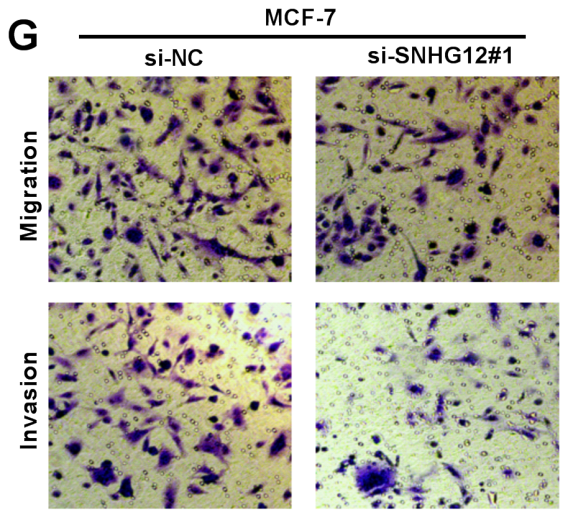

Figure 2. SNHG12 knockdown suppressed proliferation, migration, and invasion but facilitated apoptosis in BC cells. MDA-MB-231 and MCF-7 cells were transfected with si-NC, si-SNHG12\#1, si-SNHG12\#2, or si-SNHG12\#3. A) SNHG12 expression in transfected cells. B, C) Cell viability of transfected MDA-MB-231 (B) and MCF-7 (C) cells was measured by CCK-8 assay. D, E) Cell apoptosis was examined by flow cytometry. F, G) Cell migration and invasion MDA-MB-231 (F) and MCF-7 cells (G) were assessed by transwell assay. H, I) Migration (H) and invasion (I) cells number of transfected cells were counted. ${ }^{* *} \mathbf{p}<0.01$

displayed in Figure 2A, SNHG12 expression was relatively lower after SNHG12 silencing than in the control group, especially in si-SNHG12\#1 transfection group. Thus, si-SNHG12\#1 transfected cells were employed for the subsequent experiments. CCK-8 results showed that cell viability was repressed by SNHG12 silencing (Figures 2B, 2C). On the contrary, SNHG12 knockdown expedited cell apoptosis in comparison with the si-NC group (Figures 2D, $2 \mathrm{E})$. Additionally, the migrated and invaded cells numbers were reduced in MDA-MB-231 and MCF-7 cells transfected with si-SNHG12\#1 (Figures 2F-I). Taken together, SNHG12 silencing weakened the cell progression in $\mathrm{BC}$ cells.

SNHG12 acted as a sponge of miR-15a-5p. According to bioinformatics analysis by miRcode, miR- $15 \mathrm{a}-5 \mathrm{p}$ contained the potential binding sites of SNHG12 (Figure 3A). Luciferase activity was dramatically decreased in BC cells co-transfected with WT SNHG12 and miR-15a-5p mimics whereas that of MUT SNHG12 group remained unchanged, confirming the interaction between SNHG12 and miR-15a-5p (Figures 3B, $3 \mathrm{C})$. In addition, RIP assay showed that the enrichment of SNHG12 and miR-15a-5p was distinctly upregulated in BC cells with anti-Ago2 compared with anti-IgG (Figures 3D, 3E). Moreover, miR-15a-5p expression was increased after SNHG12 silencing. In the meantime, the miR-15a-5p level was decreased in BC cells transfected with SNHG12 (Figures 3F, 3G). To explore the role of miR-15a-5p, we measured the expression of miR-15a-5p in BC tumor tissues and normal tissues. As exhibited in Figure $3 \mathrm{H}$, the expression of miR-15a-5p was downregulated in BC tumor tissues compared with normal ones. By calculation, we found 
A

Position binding sites of 3'UTR

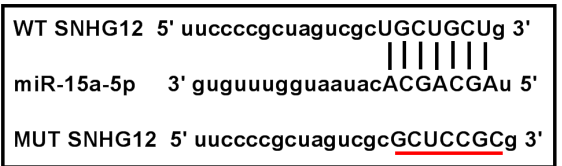

D

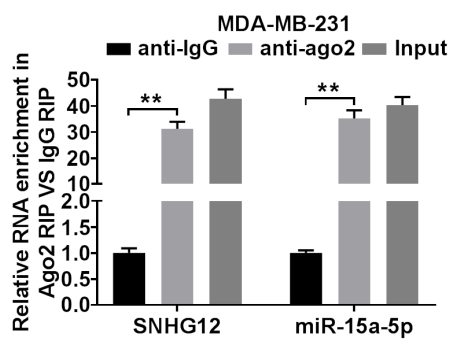

G

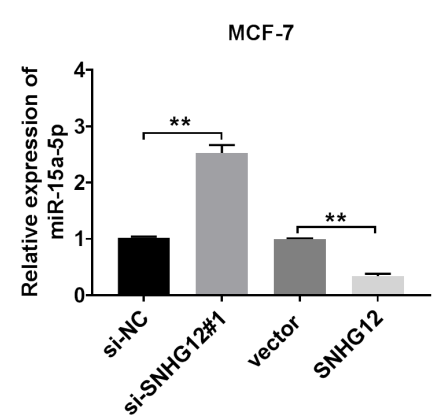

B

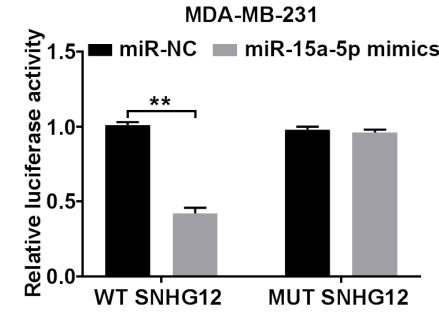

E

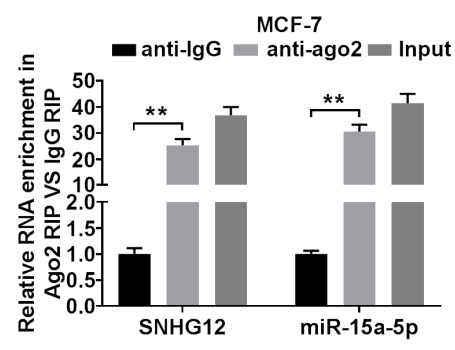

H

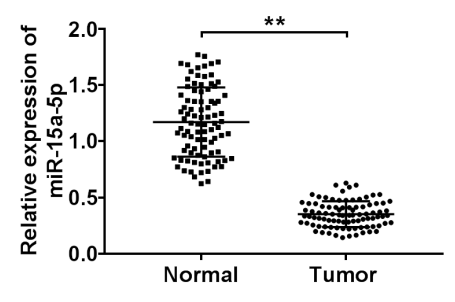

C

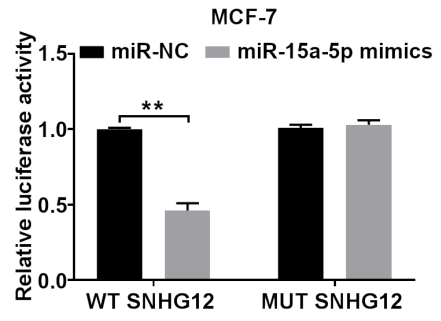

F

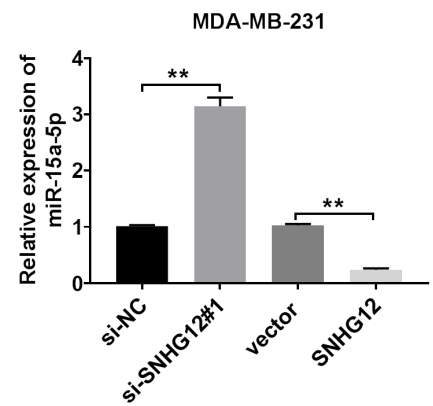

I

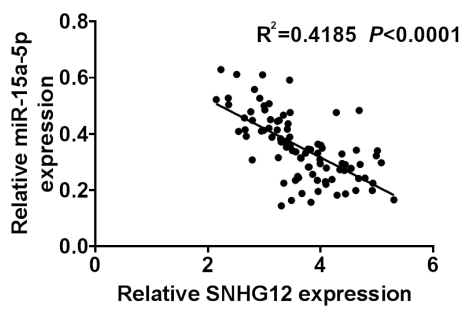

Figure 3. SNHG12 directly interacted with miR-15a-5p. A) The potential binding sites between SNHG12 and miR-15a-5p predicted by miRcode. B, C) Luciferase activity of MDA-MB-231 (B) and MCF-7 cells (C) co-transfected with WT SNHG12 or MUT SNHG7 and miR-15a-5p mimics or miR-NC. D, E) The enrichment of SNHG12 and miR-15a-5p in MDA-MB-231 (D) and MCF-7 cells (E) was detected by RIP assay. F, G) The expression of miR15a-5p in MDA-MB-231 (F) and MCF-7 cells (G) transfected with si-NC, si-SNHG12\# 1, vector, or SNHG12 was analyzed by qRT-PCR. H) The expression of miR-15a-5p in BC tumor tissues compared with the matched normal tissues. I) The correlation between SNHG12 and miR-15a-5p $\left(R^{2}=0.4185\right.$, $\mathrm{p}<0.0001) .{ }^{* *} \mathrm{p}<0.01$

that SNHG12 was negatively correlated with miR-15a-5p $\left(\mathrm{R}^{2}=0.4185, \mathrm{p}<0.0001\right.$, Figure 3I). Collectively, we concluded that SNHG12 could regulate miR-15a-5p in BC cells.

SNHG12 regulated cell proliferation, migration, invasion, and apoptosis by targeting miR-15a-5p in BC cells. To disclose the regulatory effects of SNHG12/ miR-15a-5p axis on BC cell progression, MDA-MB-231 and MCF-7 cells were transfected with si-NC, si-SNHG12\#1, si-SNHG12\#1+anti-NC or si-SNHG12\#1+anti-miR-15a-5p. As shown in Figure 4A, the expression of miR-15a-5p was enhanced by the SNHG12 silencing and repressed by the miR-15a-5p inhibitor. We also noticed that the abundance of miR-15a-5p attenuated BC cell proliferation. However, the miR-15a-5p inhibitor reversed the effects (Figures 4B, 4C). On the contrary, cell apoptosis was enhanced by SNHG12 silencing and reduced by the miR-15a-5p inhibitor (Figure 4D). Furthermore, transwell assay clarified that the
miR-15a-5p inhibitor abrogated SNHG12 silencing induced inhibition on cell migration and invasion (Figures 4E, 4F). These findings demonstrated that SNHG12 regulates BC cell growth by targeting miR-15a-5p in BC.

SALL4 was a target of miR-15a-5p. We hypothesized that miR-15a-5p regulated BC cell behavior by interacting with the target gene. Bioinformatics tool TargetScan predicted that there were binding sites between miR-15a-5p and SALL4 (Figure 5A). We also observed an apparent reduction of luciferase activity in BC cells co-transfected with WT SALL4 and miR-15a-5p mimics, validating the interaction between miR-15a-5p and SALL4 (Figures 5B, 5C). Interestingly, SALL4 protein expression was inhibited by miR-15a-5p mimics and elevated by miR-15a-5p inhibitor, implicating that SALL4 was negatively regulated by miR-15a-5p (Figure 5D). In addition, the SALL4 level was much higher in BC tumor tissues than normal tissues (Figure 5E). Person's correlation coefficient 
A

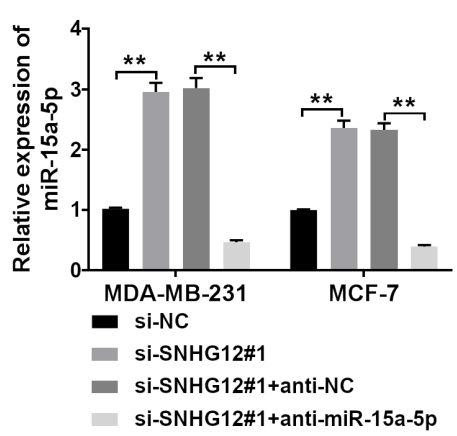

D

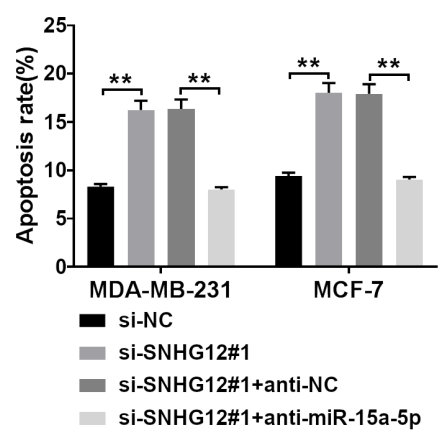

B

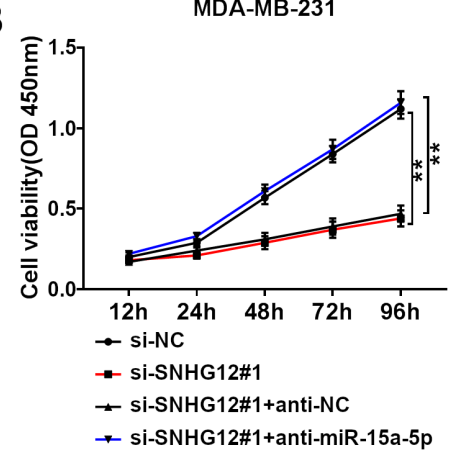

E

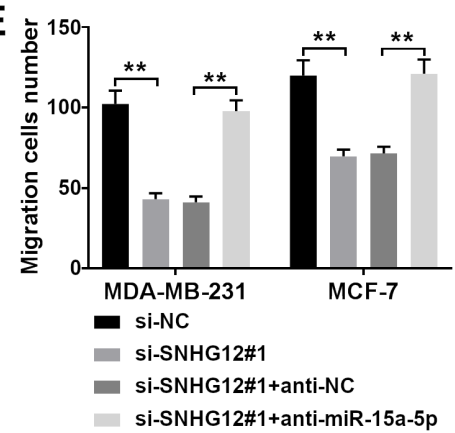

C

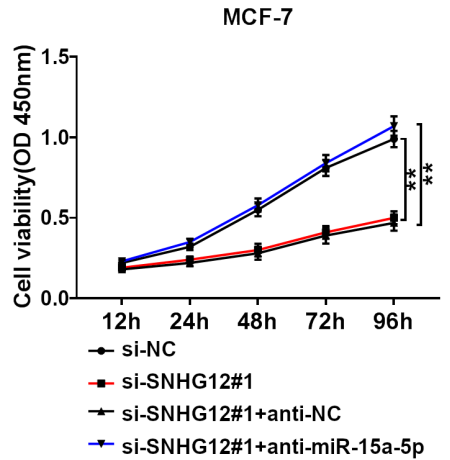

$F$

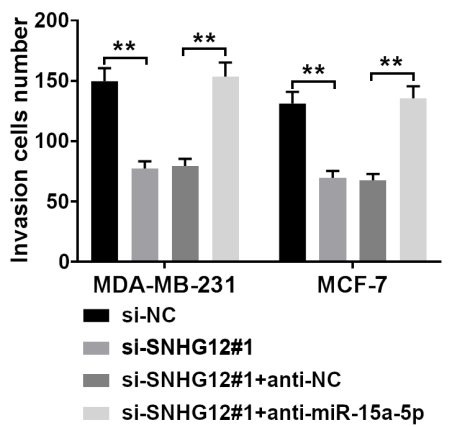

Figure 4. miR-15a-5p inhibitor attenuated SNHG12 silencing induced inhibition on BC cell proliferation, migration, and invasion. MDA-MB-231 and MCF-7 cells were transfected with si-NC, si-SNHG12\#1, si-SNHG12\#1+anti-NC, or si-SNHG12\#1+anti-miR-15a-5p. A) The expression of miR-15a-5p in transfected cells was evaluated by qRT-PCR. B, C) Cell viability of transfected MDA-MB-231 (B) and MCF-7 cells (C) was detected. D) Cell apoptosis of transfected cells was determined by flow cytometry. E, F) Cell migration (E) and invasion (F) of transfected cells were examined by transwell assay. ${ }^{* *} \mathrm{p}<0.01$

analysis results revealed that SALL4 was negatively correlated with miR-15a-5p $\left(\mathrm{R}^{2}=0.5312, \mathrm{p}<0.0001\right.$, Figure 5F). Conversely, SALL4 was positively correlated with SNHG12 $\left(R^{2}=0.4392, p<0.0001\right.$, Figure $\left.5 G\right)$. Altogether, miR-15a-5p could regulate SALL4 expression in BC cells.

SNHG12 facilitated BC cell progression by upregulating SALL4 expression via sponging miR-15a-5p. Whether SNHG12 modulated tumorigenesis and progression in $\mathrm{BC}$ by regulating the miR-15a-5p/SALL4 axis was examined in MDA-MB-231 and MCF-7 cells. Excessive expression of SALL4 protein appeared in BC cells transfected with si-SNHG12\#1+anti-miR-15a-5p and anti-miR-15a$5 p+a n t i-N C$. However, the lack of SALL4 protein expression was found in the si-SNHG12\#1+anti-NC and the antimiR-15a-5p+anti-SALL4 transfection group (Figure 6A). The upregulation of SALL4 expression was discovered to accelerate cell proliferation and suppress apoptosis in $\mathrm{BC}$ (Figures 6B, 6C). As expected, the downregulation of SALL4 expression exerted the opposite effects on $\mathrm{BC}$ cell growth (Figure 6D). Besides, migration and invasion of $B C$ cells were enhanced by increased expression of SALL4 and impaired by decreased expression of SALL4 (Figures 6E, 6F). Therefore, we suggested that SNHG12 contributed to BC cell development by regulation of the miR-15a-5p/SALL4 axis.
Interference of SNHG12 repressed tumor growth in vivo. To further validate the function of SNHG12 in vivo, the MDA-MB-231 xenograft mice model was constructed by subcutaneously injecting MDA-MB-231 cells stably transfected with sh-SNHG12 or sh-NC. As illustrated in Figures 7A and 7B, tumors grew slower in sh-SNHG12 transfection mice than that of the sh-NC group. The mice were sacrificed at day 30 , after that, all the tumors were collected and subjected to biological analysis. SNHG12 expression was decreased whereas miR-15a-5p was increased in mice after SNHG12 silencing (Figures 7C, 7D). Meanwhile, the expression of SALL4 mRNA and protein was reduced in the sh-SNHG12 group in comparison with the sh-NC group (Figures 7E, 7F). These results implied that SNHG12 silencing could repress tumor growth in vivo.

\section{Discussion}

Growing pieces of evidence have identified SNHG12 as a significant oncogene that plays promotive roles in tumorigenesis and malignancy [24, 25]. For instance, Wen et al. validated that excessive expression of SNHG12 was closely related to poor prognosis of nasopharyngeal carcinoma since SNHG12 exerted its oncogenic function to expedite 
A Position 175-181 of SALL4 3'UTR

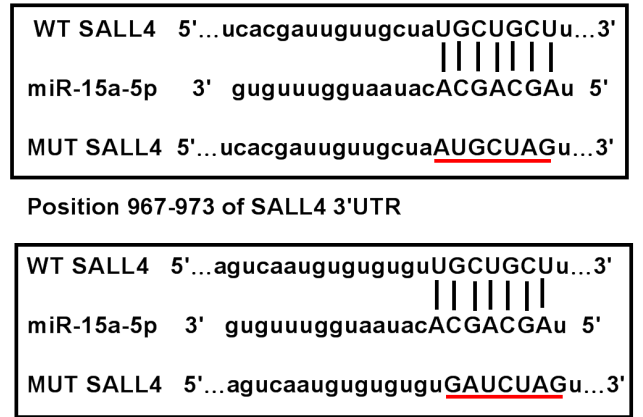

B

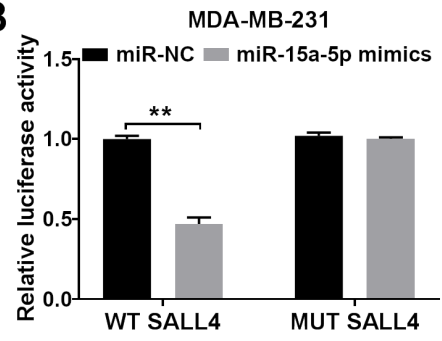

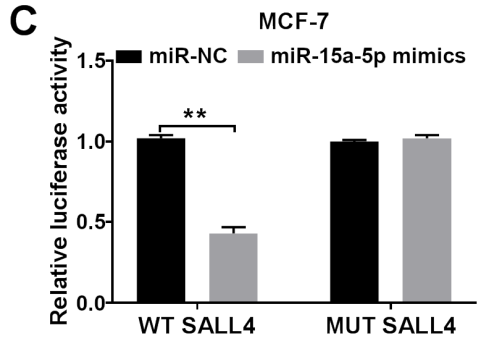

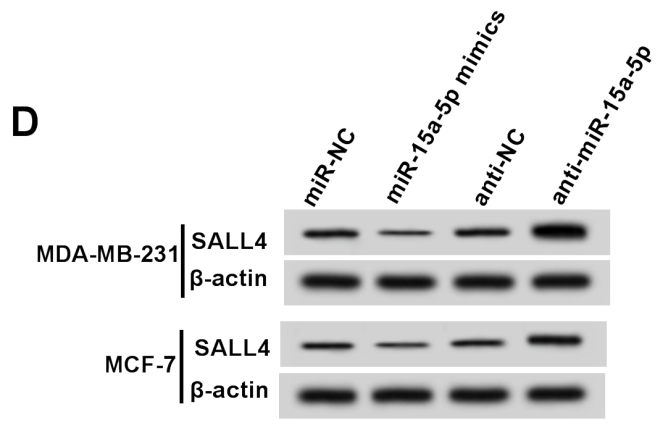

E

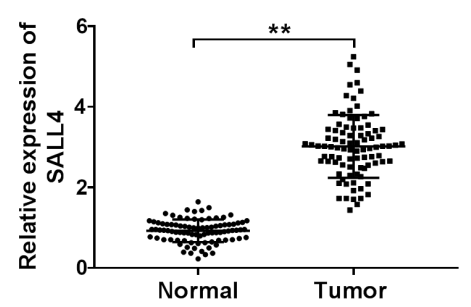

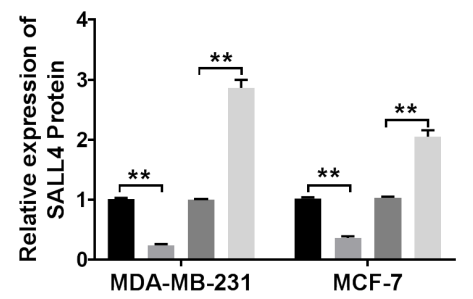

$\mathbf{F}$

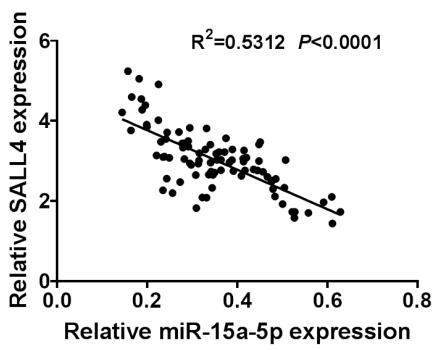

G

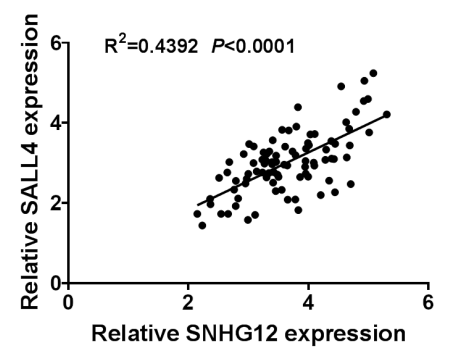

Figure 5. SALL4 was a target of miR-15a-5p. A) The potential binding sites between SALL4 and miR-15a-5p predicted by TargetScan. B, C) Luciferase activity of MDA-MB-231 (B) and MCF-7 cells (C) co-transfected with WT SALL4 or MUT SALL4 and miR-15a-5p mimics or miR-NC. D) Protein expression of SALL4 in MDA-MB-231 and MCF-7 cells transfected with miR-NC, miR-15a-5p mimics, anti-NC, and anti-miR-15a-5p. $\beta$-actin was used as an internal reference. E) SALL4 expression in BC tumor tissues and the matched normal tissues. F) The correlation between SALL4 and miR-15a-5p $\left.\left(R^{2}=0.5312, p<0.0001\right) . G\right)$ The correlation between SNHG12 and SALL4 $\left(R^{2}=0.4392, p<0.0001\right) .{ }^{* *} p<0.01$

cell proliferation and metastasis by regulating Notch signal pathway [26]. In prostate cancer, SNHG12 induced the activation of $\mathrm{Wnt} / \beta$-catenin signaling to accelerate cell proliferation by absorbing microRNA-95 [27]. Likewise, an abundance of SNHG12 promoted viability, migration, and invasion both in ovarian cancer and renal cell carcinoma cells through regulation of miRNA-129/SOX4 axis and HIF1a, respectively [28, 29]. Moreover, overexpression of SNHG12 induced by c-Myc facilitated proliferation and weakened cisplatin sensitivity in T-cell lymphoma by regulating Ki67/P-gp [30]. However, whether SNHG12 accelerated $\mathrm{BC}$ cell progression and the underlying biological mechanism is still unclear.
Bioinformatics analysis manifested that miR-15a-5p was a potential target gene of SNHG12. Interestingly, miR-15a-5p, which was associated with tumor metastasis and recurrence, has been identified as a crucial prognostic biomarker in multiple cancer types, such as colorectal adenocarcinoma, myeloid leukemia, and non-small cell lung cancer [31, 32]. Generally, miR-15a-5p functioned as a tumor suppressor to repress cancer cell growth. For instance, miR-15a-5p was downregulated in human hepatocellular carcinoma and increased expression of miR-15a-5p suppressed cell viability and division by enhancing brain-derived neurotrophic factor (BDNF) level [33]. Gao et al. found that miR-15a-5p alleviated chronic myeloid leukemia cell survival and metastasis by 
A

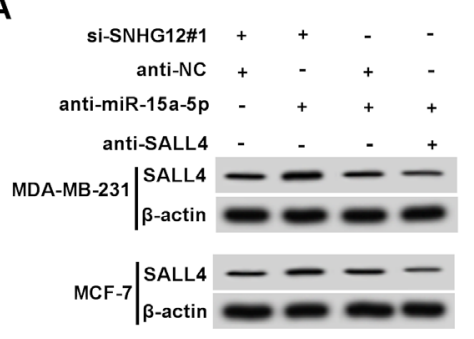

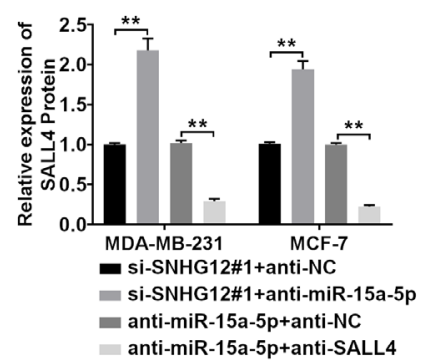

B

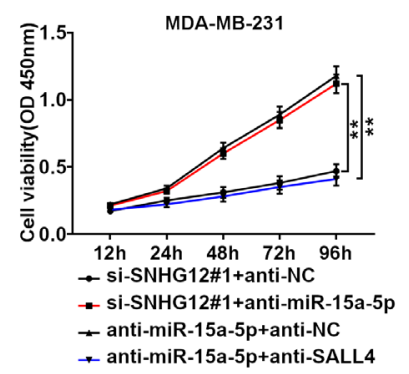

C

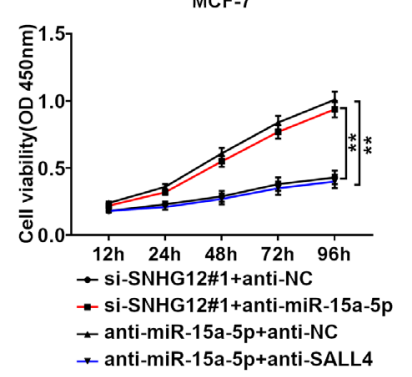

D

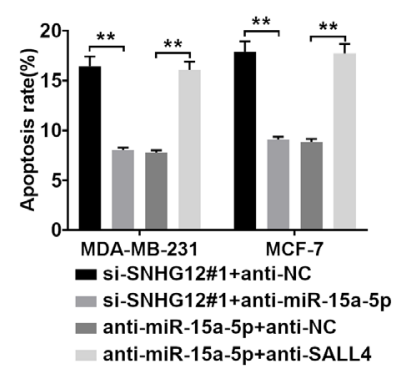

E

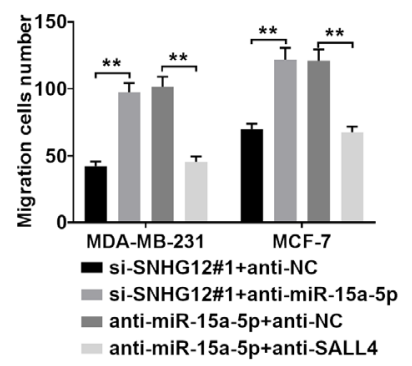

$\mathrm{F}$

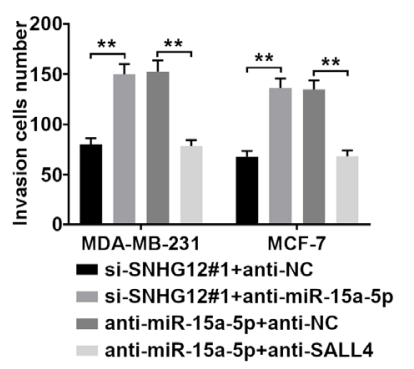

Figure 6. SNHG12 affected cell proliferation, migration, invasion, and apoptosis in BC cells by regulating miR-15a-5p/SALL4 axis. MDA-MB-231 and MCF-7 cells were transfected with si-SNHG12\#1+anti-NC, si-SNHG12\#1+anti-miR-15a-5p, anti-miR-15a-5p+anti-NC, or anti-miR-15a-5p+antiSALL4. A) Protein expression of SALL4 in transfected cells. $\beta$-actin was used as an internal reference. B, C) Cell viability of transfected MDA-MB-231 (B) and MCF-7 cells (C) was measured. D) Cell apoptosis of transfected cells was measured. E, F) Cell migration (E) and invasion (F) of transfected cells were determined by transwell assay. ${ }^{* *} \mathrm{p}<0.01$

A
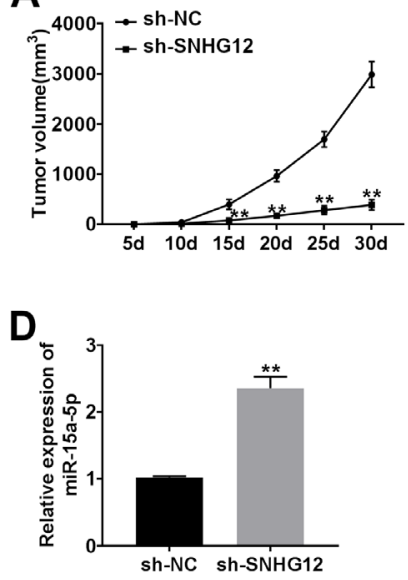

B

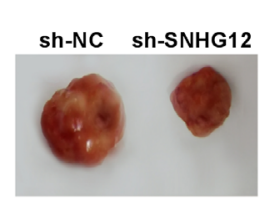

E

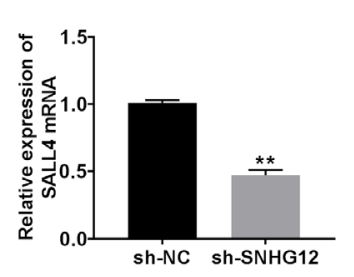

C
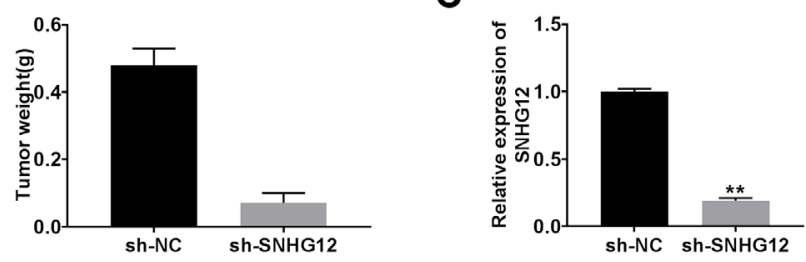

$\mathbf{F}$

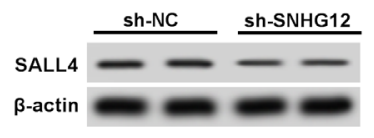

Figure 7. SNHG12 knockdown inhibited tumor growth in vivo. MDA-MB-231 xenograft mice were established by subcutaneously injecting MDAMB-231 cells stably transfected with sh-SNHG12 and sh-NC. A, B) Tumor volume (A) and weight (B) were measured. C, D) SNHG12 (C) and miR-15a$5 p$ (D) expression in tumor tissues harvested from xenograft mice. E, F) The expression of SALL4 mRNA (E) and protein (F) in tumors was detected by $\mathrm{qRT}-\mathrm{PCR}$ and western blot. ${ }^{* *} \mathrm{p}<0.01$

modulating the target gene chemokine ligand 10 (CXCL10) [34]. On the contrary, the abundance of miR-15a-5p was clarified as one of the pathogenic factors of the abdominal aortic aneurysm by interfering cyclin dependent kinase inhibitor $2 \mathrm{~B}$ [35]. However, the molecular mechanism of miR-15a-5p in $\mathrm{BC}$ cell regulation requires further exploration.
SALL4 was predicted as a target of miR-15a-5p using TargetScan. Previous studies indicated that SALL4 was implicated in multiple cancers. For instance, SALL4 was reported to regulate CTNNB1 expression and further contribute to tumorigenicity and progression in cervical cancer via altering the Wnt/ $\beta$-catenin pathway [36]. Consistently, SALL4 accel- 
erated the malignancy of gastric cancer by promoting cell metastasis and epithelial to mesenchymal transition (EMT) processes through TGF- $\beta /$ SMAD pathway [37]. Therefore, we suggested that the target gene SALL4 played a promotive role in $\mathrm{BC}$ growth.

A series of experiments were employed to reveal the biological and pathological mechanisms of BC. The expressions of SNHG12 and SALL4 were upregulated while miR-15a-5p was downregulated in BC tumor tissues compared with the corresponding normal tissues. Loss of function experiments revealed that SNHG12 silencing hindered cell growth in vitro and in vivo. In addition, the luciferase reporter system results confirmed the interaction between miR-15a-5p and SNHG12 or SALL4. Person's correlation coefficient analysis showed that miR-15a-5p was correlated with SNHG12 and SALL4 inversely. The subsequent rescue experiments indicated that miR-15a-5p inhibitor restored SNHG12 silencing mediated inhibition on cell viability, migration, and invasion as well as promotion on apoptosis in BC. More importantly, we discovered that SNHG12 mediated cell regulation through absorbing miR-15a-5p to elevate SALL4 expression.

In conclusion, our study demonstrated that SNHG12 accelerated proliferation, migration, and invasion whereas repressed apoptosis by upregulating SALL4 expression via interacting with miR-15a-5p, representing promising targeted therapy strategy for BC.

\section{References}

[1] DALTON SO, SUPPLI NP, EWERTZ M, KROMAN N, GRASSI L et al. Impact of schizophrenia and related disorders on mortality from breast cancer: a population-based cohort study in Denmark, 1995-2011. Breast 2018; 40: 170176. https://doi.org/10.1016/j.breast.2018.06.002

[2] BURT LM, YING J, POPPE MM, SUNEJA G, GAFFNEY DK. Risk of secondary malignancies after radiation therapy for breast cancer: comprehensive results. Breast 2017; 35: 122-129. https://doi.org/10.1016/j.breast.2017.07.004

[3] LJEPOJA B, GARCIA-ROMAN J, SOMMER AK, WAGNER E, ROIDL A. MiRNA-27a sensitizes breast cancer cells to treatment with Selective Estrogen Receptor Modulators. Breast 2019; 43: 31-38. https://doi.org/10.1016/j. breast.2018.10.007

[4] PEREZ-GARCIA J, MUNOZ-COUSELO E, SOBERINO J, RACCA F, CORTES J. Targeting FGFR pathway in breast cancer. Breast 2018; 37: 126-133. https://doi.org/10.1016/j. breast.2017.10.014

[5] NAZARI SS, MUKHERJEE P. An overview of mammographic density and its association with breast cancer. Breast Cancer 2018; 25: 259-267. https://doi.org/10.1007/s12282018-0857-5

[6] SPRONK I, SCHELLEVIS FG, BURGERS JS, DE BOCK $\mathrm{GH}, \mathrm{KOREVAAR} \mathrm{JC.} \mathrm{Incidence} \mathrm{of} \mathrm{isolated} \mathrm{local} \mathrm{breast} \mathrm{can-}$ cer recurrence and contralateral breast cancer: a systematic review. Breast 2018; 39: 70-79. https://doi.org/10.1016/j. breast.2018.03.011
[7] SMITH CR, CHIARELLI AM, HOLLOWAY CM, MIREA L, O'MALLEY FP et al. The impact of organized breast assessment on survival by stage for screened women diagnosed with invasive breast cancer. Breast 2018; 41: 25-33. https:// doi.org/10.1016/j.breast.2018.06.007

[8] ZHANG S, MA H, ZHANG D, XIE S, WANG W et al. LncRNA KCNQ1OT1 regulates proliferation and cisplatin resistance in tongue cancer via miR-211-5p mediated Ezrin/ Fak/Src signaling. Cell Death Dis 2018; 9: 742. https://doi. org/10.1038/s41419-018-0793-5

[9] ZHU TT, SUN RL, YIN YL, QUAN JP, SONG P et al. Long noncoding RNA UCA1 promotes the proliferation of hypoxic human pulmonary artery smooth muscle cells. Pflugers Arch 2019; 471: 347-355. https://doi.org/10.1007/s00424018-2219-8

[10] MA H, SU R, FENG H, GUO Y, SU G. Long noncoding RNA UCA1 promotes osteosarcoma metastasis through CREB1mediated epithelial-mesenchymal transition and activating PI3K/AKT/mTOR pathway. J Bone Oncol 2019; 16: 100228. https://doi.org/10.1016/j.jbo.2019.100228

[11] FENG W, WANG C, LIANG C, YANG H, CHEN D et al. The dysregulated expression of KCNQ1OT1 and Its Interaction with downstream factors miR-145/CCNE2 in breast cancer cells. Cell Physiol Biochem 2018; 49: 432-446. https://doi. org/10.1159/000492978

[12] XU M, CHEN X, LIN K, ZENG K, LIU X et al. The long noncoding RNA SNHG1 regulates colorectal cancer cell growth through interactions with EZH2 and miR-154-5p. Mol Cancer 2018; 17: 141. https://doi.org/10.1186/s12943018-0894-X

[13] YIN WL, YIN WG, HUANG BS, WU LX. LncRNA SNHG12 inhibits miR-199a to upregulate SIRT1 to attenuate cerebral ischemia/reperfusion injury through activating AMPK signaling pathway. Neurosci Lett 2019; 690: 188-195. https:// doi.org/10.1016/j.neulet.2018.08.026

[14] YAO X, YAO R, HUANG F, YI J. LncRNA SNHG12 as a potent autophagy inducer exerts neuroprotective effects against cerebral ischemia/reperfusion injury. Biochem Biophys Res Commun 2019; 514: 490-496. https://doi.org/10.1016/j. bbrc.2019.04.158

[15] ZHAO M, WANG J, XI X, TAN N, ZHANG L. SNHG12 promotes angiogenesis following ischemic stroke via regulating miR-150/VEGF pathway. Neuroscience 2018; 390: 231-240. https://doi.org/10.1016/j.neuroscience.2018.08.029

[16] ZHAO D, SUI Y, ZHENG X. MiR-331-3p inhibits proliferation and promotes apoptosis by targeting HER2 through the PI3K/Akt and ERK1/2 pathways in colorectal cancer. Oncol Rep 2016; 35: 1075-1082. https://doi.org/10.3892/ or.2015.4450

[17] ZHANG Y, CAI H, CHEN S, SUN D, ZHANG D et al. Exosomal transfer of miR-124 inhibits normal fibroblasts to cancer-associated fibroblasts transition by targeting sphingosine kinase 1 in ovarian cancer. J Cell Biochem 2019. https://doi. org/10.1002/jcb.28593

[18] YIN Y, ZHANG B, WANG W, FEI B, QUAN C et al. MiR204-5p inhibits proliferation and invasion and enhances chemotherapeutic sensitivity of colorectal cancer cells by downregulating RAB22A. Clin Cancer Res 2014; 20: 6187-6199. https://doi.org/10.1158/1078-0432.CCR-14-1030 
[19] DUAN ZX, HUANG P, TU C, LIU Q, LI SQ et al. MicroRNA-15a-5p regulates the development of osteoarthritis by targeting PTHrP in chondrocytes. Biomed Res Int 2019; 2019: 3904923. https://doi.org/10.1155/2019/3904923

[20] DIETER C, ASSMANN TS, COSTA AR, CANANI LH, DE SOUZA BM et al. MiR-30e-5p and mir-15a-5p expressions in plasma and urine of Type 1 diabetic patients with diabetic kidney disease. Front Genet 2019; 10: 563. https://doi. org/10.3389/fgene.2019.00563

[21] JIANG L, WU Z, MENG X, CHU X, HUANG H et al. LncRNA HOXA-AS2 facilitates tumorigenesis and progression of papillary thyroid cancer by modulating the miR-15a-5p/ HOXA3 Axis. Hum Gene Ther 2019; 30: 618-631. https:// doi.org/10.1089/hum.2018.109

[22] LIU Y, LIU LY, JIA Y, SUN YY, MA FZ. Role of microRNA$15 a-5 p$ in the atherosclerotic inflammatory response and arterial injury improvement of diabetic by targeting FASN. Biosci Rep 2019; 39. https://doi.org/10.1042/BSR20181852

[23] CHEN H, TIAN Y. MiR-15a-5p regulates viability and matrix degradation of human osteoarthritis chondrocytes via targeting VEGFA. Biosci Trends 2017; 10: 482-488. https:// doi.org/10.5582/bst.2016.01187

[24] JIN XJ, CHEN XJ, ZHANG ZF, HU WS, OU RY et al. Long noncoding RNA SNHG12 promotes the progression of cervical cancer via modulating miR-125b/STAT3 axis. J Cell Physiol 2019; 234: 6624-6632. https://doi.org/10.1002/ jcp. 27403

[25] SUN Y, LIU J, CHU L, YANG W, LIU H et al. Long noncoding RNA SNHG12 facilitates the tumorigenesis of glioma through miR-101-3p/FOXP1 axis. Gene 2018; 676: 315-321. https://doi.org/10.1016/j.gene.2018.08.034

[26] LIU ZB, TANG C, JIN X, LIU SH, PI W. Increased expression of lncRNA SNHG12 predicts a poor prognosis of nasopharyngeal carcinoma and regulates cell proliferation and metastasis by modulating Notch signal pathway. Cancer Biomark 2018; 23: 603-613. https://doi.org/10.3233/CBM181873

[27] SONG J, WU X, MA R, MIAO L, XIONG L et al. Long noncoding RNA SNHG12 promotes cell proliferation and activates $\mathrm{Wnt} /$ beta-catenin signaling in prostate cancer through sponging microRNA-195. J Cell Biochem 2019; 120: $13066-$ 13075. https://doi.org/10.1002/jcb.28578
[28] CHEN Q, ZHOU W, DU SQ, GONG DX, LI J et al. Overexpression of SNHG12 regulates the viability and invasion of renal cell carcinoma cells through modulation of HIF1alpha. Cancer Cell Int 2019; 19: 128. https://doi.org/10.1186/ s12935-019-0782-5

[29] SUN D, FAN XH. LncRNA SNHG12 accelerates the progression of ovarian cancer via absorbing miRNA-129 to upregulate SOX4. Eur Rev Med Pharmacol Sci 2019; 23: 2345-2352. https://doi.org/10.26355/eurrev_201903_17378

[30] ZHU L, ZHANG X, FU X, LI Z, SUN Z et al. c-Myc mediated upregulation of long noncoding RNA SNHG12 regulates proliferation and drug sensitivity in natural killer/T-cell lymphoma. J Cell Biochem 2019; 120: 12628-12637. https:// doi.org/10.1002/jcb.28529

[31] KONTOS CK, TSIAKANIKAS P, AVGERIS M, PAPADOPOULOS IN, SCORILAS A. MiR-15a-5p, a novel prognostic biomarker, predicting recurrent colorectal adenocarcinoma. Mol Diagn Ther 2017; 21: 453-464. https://doi.org/10.1007/ s40291-017-0270-3

[32] ERGUN S, GÜNEY S, TEMIZ E, PETROVIC N, GUNES S. Significance of miR-15a-5p and CNKSR3 as novel prognostic biomarkers in non-small cell lung cancer. Anticancer Agents Med Chem 2018; 18: 1695-1701. https://doi.org/10.2 174/1871520618666180718100656

[33] LONG J, JIANG C, LIU B, FANG S, KUANG M. MicroRNA$15 a-5 p$ suppresses cancer proliferation and division in human hepatocellular carcinoma by targeting BDNF. Tumour Biol 2016; 37: 5821-5828. https://doi.org/10.1007/s13277015-4427-6

[34] DAN CHEN1 DW. MiR-15a-5p negatively regulates cell survival and metastasis by targeting CXCL10 in chronic myeloid leukemia. Am J Transl Res 2017; 9: 4308-4316.

[35] GAO P, SI J, YANG B, YU J. Upregulation of microRNA-15a contributes to pathogenesis of abdominal aortic aneurysm (AAA) by modulating the expression of cyclin-dependent kinase inhibitor 2B (CDKN2B). Med Sci Monit 2017; 23: 881-888. https://doi.org/10.12659/msm.898233

[36] CHEN M, LI L, ZHENG PS. SALL4 promotes the tumorigenicity of cervical cancer cells through activation of the Wnt/ $\beta$-catenin pathway via CTNNB1. Cancer Sci 2019; 110: 2794-2805. https://doi.org/10.1111/cas.14140

[37] ZHANG X, ZHANG P, SHAO $\mathrm{M}$ et al. SALL4 activates TGF- $\beta /$ SMAD signaling pathway to induce EMT and promote gastric cancer metastasis. Cancer Manag Res 2018; 10: 4459-4470. https://doi.org/10.2147/CMAR.S177373 